\title{
Graphitic- $\mathrm{C}_{3} \mathrm{~N}_{4} \quad$ hybridized $\quad \mathrm{N}$-doped $\quad \mathrm{La}_{2} \mathrm{Ti}_{2} \mathrm{O}_{7}$ two-dimensional layered composites as efficient visible-light-driven photocatalyst
}

Xiaoyan Cai ${ }^{\mathrm{a}, \mathrm{b}}$, Junying Zhang ${ }^{\mathrm{a}, *}$, Mamoru Fujitsuka ${ }^{\mathrm{b}}$, and Tetsuro Majima ${ }^{\mathrm{b}, *}$

a Key Laboratory of Micro-nano Measurement, Manipulation and Physics (Ministry of Education), Department of Physics, Beihang University, Beijing 100191, P. R. China

b The Institute of Scientific and Industrial Research (SANKEN), Osaka University, Mihogaoka 8-1, Ibaraki, Osaka 567-0047, Japan

*Corresponding authors. E-mail: zjy@ buaa.edu.cn (J. Zhang); majima@ sanken.osaka-u.ac.jp (T. Majima). 


\section{ABSTRACT}

Perovskite-type $\mathrm{La}_{2} \mathrm{Ti}_{2} \mathrm{O}_{7}$ (LTO), having a layered structure and the separated $\mathrm{H}_{2}$ and $\mathrm{O}_{2}$ evolution sites, is attractive as an efficient photocatalyst. However, the photocatalytic activity is often limited by the poor electron mobility. This problem can be conquered by hybridization with materials having efficient properties for the visible light absorption and charge carrier transport. Here, we report a two-dimensional (2D) layered composite hybridized by approximately $2 \mathrm{~nm}$ thick graphitic $\mathrm{C}_{3} \mathrm{~N}_{4}$ nanosheets $\left(\mathrm{g}-\mathrm{C}_{3} \mathrm{~N}_{4}\right)$ and $7 \mathrm{~nm}$ thick nitrogen doped LTO nanosheets (NLTO) $\left(\mathrm{g}-\mathrm{C}_{3} \mathrm{~N}_{4} / \mathrm{NLTO}\right)$, in which $\mathrm{g}-\mathrm{C}_{3} \mathrm{~N}_{4}$ and NLTO act as hole receptor and electron conductor, respectively. g- $\mathrm{C}_{3} \mathrm{~N}_{4} / \mathrm{NLTO}$ exhibited high photocatalytic activities for $\mathrm{H}_{2}$ production via water splitting and dye degradation under the UV and visible light irradiation, due to the interfacial charge transfer at the interface between $g-\mathrm{C}_{3} \mathrm{~N}_{4}$ and NLTO. The electrochemical measurement showed the type II band alignment with favorable charge transfer from $\mathrm{g}-\mathrm{C}_{3} \mathrm{~N}_{4}$ to NLTO. The $2 \mathrm{D}$ architecture with a maximized interfacial area allows efficient charge separation with a short interfacial distance. This efficient interfacial charge transfer is further elucidated from monitoring of charge separation and trapping processes using the femtosecond time-resolved diffused reflectance (TDR) measurement.

keywords

Nitrogen doped $\mathrm{La}_{2} \mathrm{Ti}_{2} \mathrm{O}_{7}, \mathrm{~g}-\mathrm{C}_{3} \mathrm{~N}_{4}$, Layered composites, Charge transfer dynamics 


\section{Introduction}

Semiconductor photocatalysts have attracted considerable attention from scientists and engineers owing to their broad potential applications in solar energy conversion, medical photodynamic therapy, and environmental remediation [1]. Of the numerous photocatalysts, layer-structured semiconductors have received extensive attention owing to their layered structures which separate out the $\mathrm{H}_{2}$ and $\mathrm{O}_{2}$ evolution sites [2-4]. Perovskite-type photocatalysts consist of the network of corner-sharing octahedra with efficient mobility of charge carriers, showing high photocatalytic activity [5-7]. Perovskite-type $\mathrm{La}_{2} \mathrm{Ti}_{2} \mathrm{O}_{7}$ (LTO) with a layered structure possesses the merits of both perovskite and layered structures to exhibit high stability and photocatalytic activity for organic decomposition, water splitting, and $\mathrm{CO}_{2}$ reduction under the ultraviolet (UV) light irradiation [8-10]. However, the wide band gap (3.0-4.0 eV) limits the absorption spectral range within the UV light region. In addition, the ionic character at room temperature causes the poor electron mobility and short charge carrier lifetime $[11,12]$.

In the present paper, such problems of LTO as the photocatalyst are resolved by materials engineering to induce efficient solar energy harvesting and generation of charge carrier with longer lifetime. The recent experimental results conducted by Wu et al. demonstrated that the N-doped LTO (NLTO) nanosheets fabricated by subsequent annealing treatment in ammonia enhanced photocatalytic activity under the visible and UV irradiation $[8,12,13]$. Considerable reports have been focused on $\mathrm{N}$-doping 
semiconductor thin films and powders prepared by annealing at high temperature under $\mathrm{NH}_{3}$ flow. The $\mathrm{N}$-doping method generally requires tail gas treatment and results in small amounts of N-loading $(<2.5 \mathrm{~mol} \%)$ [14-17]. Here, we present a hydrothermal method to prepare NLTO nanosheets which is simple, inexpensive, and able to produce large quantities of nanomaterials with larger amounts of $\mathrm{N}$-loading $(>2.5$ $\mathrm{mol} \%)$ at lower temperature $\left(<230^{\circ} \mathrm{C}\right)$. To our best knowledge, this is the first time to employ the hydrothermal method to dope $\mathrm{N}$ into layered perovskite photocatalysts. Furthermore, graphitic $\mathrm{C}_{3} \mathrm{~N}_{4}\left(\mathrm{~g}-\mathrm{C}_{3} \mathrm{~N}_{4}\right)$ was hybridized with NLTO nanosheets to further enhance the charge separation. $\mathrm{g}-\mathrm{C}_{3} \mathrm{~N}_{4}$ has high thermal and chemical stability, and has been widely studied in the field of photocatalysis in recent years [18-20]. It exhibits a stacking 2D layered structure, similar to that of graphite [21]. Hybridizing two kinds of 2D layered materials greatly enhances the photocatalytic activity due to increasing the interface area and interfacial charge transfer rate [22-24]. As a result, 2D layered composite hybridized by $\mathrm{g}-\mathrm{C}_{3} \mathrm{~N}_{4}$ and NLTO $\left(\mathrm{g}-\mathrm{C}_{3} \mathrm{~N}_{4} / \mathrm{NLTO}\right)$ is expected to show high photocatalytic activity.

In this study we present an approach for maximized interfacial area and shortened charge transport time and distance using ultrathin $\mathrm{g}-\mathrm{C}_{3} \mathrm{~N}_{4}$ nanosheets as a hole receptor and NLTO nanosheets as an electron conductor. This 2D heterostructure allows for simultaneously high visible light absorption and carrier collection efficiency. We also experimentally demonstrate thermodynamically favorable band alignment between NLTO and $\mathrm{g}-\mathrm{C}_{3} \mathrm{~N}_{4}$ nanosheets using electrochemical measurement. 
In addition, the interfacial charge transfer mechanism between NLTO and $\mathrm{g}-\mathrm{C}_{3} \mathrm{~N}_{4}$ was explored by femtosecond time-resolved diffuse reflection spectral measurement.

\section{Experimental}

\subsection{Preparation of LTO nanosheets}

LTO nanosheets were synthesized through one-step hydrothermal method as shown in Scheme $\mathrm{S} 1$ (a). $2 \mathrm{mmol}$ of $\mathrm{La}\left(\mathrm{NO}_{3}\right)_{3} \cdot 6 \mathrm{H}_{2} \mathrm{O}$ (Wako Pure Chemical Industries, Ltd.) and $2 \mathrm{mmol}$ of $\mathrm{Ti}\left(\mathrm{SO}_{4}\right)_{2}$ (Wako, 30\% aqueous) were dissolved in $10 \mathrm{~mL}$ Milli-Q ultrapure water (Millipore). $10 \mathrm{~mL} \mathrm{NaOH}$ solution (Wako, $2 \mathrm{M}$ ) was dropped into the above reaction solution. After magnetically stirring for $4 \mathrm{~h}$, the mixture was poured into a Teflon cup $(50 \mathrm{~mL})$ in a stainless autoclave and then heat at $230{ }^{\circ} \mathrm{C}$ for $24 \mathrm{~h}$. After the completion of reaction, the autoclave was taken out from the incubator and left to cool-down spontaneously to room temperature. The white precipitates were collected after centrifugation, washing and drying in air at $80{ }^{\circ} \mathrm{C}$ for $12 \mathrm{~h}$ [8].

\subsection{Preparation of NLTO}

Nitrogen was doped into LTO from the hydrothermal method, followed by acid treatment to remove the byproduct (Scheme $\mathrm{S} 1(\mathrm{~b})$ ). $0.3 \mathrm{~g}$ of prepared LTO white powders were added into $30 \mathrm{~mL}$ triethanolamine (TEOA) (Sigma-Aldrich) aqueous solution. After stirring for $1 \mathrm{~h}$, the solution was put into a Teflon-lined stainless autoclave and heated at $220{ }^{\circ} \mathrm{C}$ for $48 \mathrm{~h}$. The resulting solution was cooled down to room temperature. Then, it was centrifuged and washed by Milli-Q ultrapure water (Millipore) and absolute ethanol (Nacalai Tesque, Inc.) for several times. To remove 
the byproduct of $\mathrm{LaCO}_{3} \mathrm{OH}$, the obtained dark yellow precipitates were dispersed in $0.1 \mathrm{M} \mathrm{HNO}_{3}$ (Wako, 69\%aqueous) solution. After stirring for $30 \mathrm{~min}$, the resultant bright yellow precipitates were collected after centrifugation, washing and drying in air at $80{ }^{\circ} \mathrm{C}$ for $12 \mathrm{~h}$.

\subsection{Preparation of $g-C_{3} N_{4}$ nanosheets}

Bulk g- $\mathrm{C}_{3} \mathrm{~N}_{4}$ was synthesized by direct heating dicyandiamide (Aldrich) at $550{ }^{\circ} \mathrm{C}$ for $4 \mathrm{~h}$ in static air. The resultant yellow agglomerates were milled into powder in a mortar. The light yellow powder of $\mathrm{g}_{-} \mathrm{C}_{3} \mathrm{~N}_{4}$ nanosheets were prepared by thermal oxidation etching of bulk $\mathrm{g}-\mathrm{C}_{3} \mathrm{~N}_{4}$ obtained as above in static air as follows: $400 \mathrm{mg}$ of bulk $\mathrm{g}-\mathrm{C}_{3} \mathrm{~N}_{4}$ was placed in an open ceramic container and heated at $500{ }^{\circ} \mathrm{C}$ for $2 \mathrm{~h}[20]$.

\subsection{Preparation of $g-\mathrm{C}_{3} \mathrm{~N}_{4} / \mathrm{NLTO}$}

$0.3 \mathrm{~g}$ of as-prepared NLTO was dispersed into $20 \mathrm{~mL}$ Milli-Q ultrapure water (Millipore). Then, $1 \mathrm{~mL}$ of bulk $\mathrm{g}-\mathrm{C}_{3} \mathrm{~N}_{4}$ dispersion with different concentration was added dropwise into the N-LTO solution under magnetic stirring to form a mixture. After stirring for $1 \mathrm{~h}$ and ultrasonic dispersion for $2 \mathrm{~h}$, the mixture was dried in an oven at $85{ }^{\circ} \mathrm{C}$ for $12 \mathrm{~h}$ to evaporate the solvents. The obtained sample was ground and heated at $500{ }^{\circ} \mathrm{C}$ for $2 \mathrm{~h}(\mathrm{Scheme} \mathrm{S} 1(\mathrm{c}))$.

\subsection{Characterization}

The structures of the samples were examined by X-ray diffraction (XRD, Rigaku, Smartlab; operated at $40 \mathrm{kV}$ and $200 \mathrm{~mA}, \mathrm{Cu} \mathrm{K \alpha}$ source). The morphologies were investigated using field-emission scanning electron microscopy (FESEM; JEOL, 
JSM-6330FT) equipped with EDX analyzer and transmission electron microscopy (TEM; JEOL, JEM3000F operated at $300 \mathrm{kV}$ or Hitachi, H-800 operated at $200 \mathrm{kV}$ ). Chemical compositions and valence band spectra of the samples were analyzed using X-ray photoelectron spectroscopy (XPS, Shimadzu AXIS-165x). The thicknesses of the samples were measured using atomic force microscopy (AFM, Seiko Instruments Inc., SPA400). The steady-state UV-Vis absorption and diffuse reflectance spectra were measured by UV-Vis-NIR spectrophotometers (Jasco, V-770) at room temperature.

\subsection{Photocatalytic degradation}

For typical photocatalytic runs, $10 \mathrm{~mL}$ of $\mathrm{g}_{-} \mathrm{C}_{3} \mathrm{~N}_{4} / \mathrm{NLTO}$ dispersion $\left(1.0 \mathrm{~g} \mathrm{~L}^{-1}\right)$ containing methyl orange (MO, Sigma-Aldrich) aqueous solution $\left(10 \mathrm{mg} \mathrm{L}^{-1}\right)$ was sonicated for $30 \mathrm{~s}$. In order to reach complete adsorption/desorption equilibration, the reaction solution was stirred under dark condition for $1 \mathrm{~h}$. The photocatalytic reaction was carried out by using a Xe light source (Asahi Spectra, LAX-C100; $500 \mathrm{~mW} \mathrm{~cm}{ }^{-2}$ ) through the filters $(\lambda>400 \mathrm{~nm}$ ) and a UV-LED source (Asahi Spectra, POT-365; 100 $\mathrm{mW} \mathrm{cm}^{-2}$ ) at room temperature, respectively. During the illumination, $1 \mathrm{~mL}$ of the sample was centrifuged at $10000 \mathrm{rpm}$ (Hitachi, himac CF16RX) to remove the particles. The concentration of unreacted molecules was analyzed by a UV-vis spectrophotometer (Shimadzu, UV-3100) at the characteristic wavelength, from which the degradation yield was calculated.

\subsection{Photocatalytic $\mathrm{H}_{2}$ generation}

$2.0 \mathrm{wt} \%$ of Pt co-catalyst was loaded on $\mathrm{g}-\mathrm{C}_{3} \mathrm{~N}_{4} / \mathrm{NLTO}$ by the photoreduction 
method. The photocatalyst (5 mg) was suspended in $5 \mathrm{~mL}$ water (Millipore) with $20 \mathrm{vol} \%$ methanol (Nacalai Tesque) in $30 \mathrm{~mL}$ glass tube. Then, the tube was sealed with a rubber septum and purged with Ar gas for 20 min before initiating the irradiation. The sample was irradiated with a Xe light source (Asahi Spectra, LAX-C100; $500 \mathrm{~mW} \mathrm{~cm}^{-2}$ ) with constant magnetic stirring at room temperature. After the reaction, $0.1 \mathrm{~mL}$ of gas was collected from the headspace of the reactor and analyzed using a gas chromatograph (Shimadzu GC-8A) equipped with a column and thermal conductivity detector. The apparent quantum efficiency (AQE) for $\mathrm{H}_{2}$ evolution at each centered wavelength of the monochromatic light with width of $\pm 5 \mathrm{~nm}$ was calculated from the following equation: $\mathrm{AQE}=\left(2 \times\right.$ number of produced $\mathrm{H}_{2} /$ number of absorbed photons $) \times 100 \%$.

\subsection{Photoelectrochemical measurements}

$10 \mathrm{mg}$ of photocatalyst powder was mixed with $20 \mu \mathrm{L}$ water (Millipore), $20 \mu \mathrm{L}$ ethanol (Nacalai Tesque) and $10 \mu \mathrm{L}$ nafion (Aldrich, 5 wt.\%) under ultrasound. The formed homogenous suspension was pasted onto an ITO substrate (BAS Inc., ITO11) with doctor blading method. The pasted ITO photoelectrode was then dried on a hot plate in air. A three-electrode photoelectrochemical cell (PEC) was constructed and measured using an electrochemical analyzer (ALS, model 660A). The Pt wire, $\mathrm{Ag} / \mathrm{AgCl}$, and photoelectrodes were used as the counter electrode, the reference electrode, and the working electrode, respectively. $0.1 \mathrm{M} \mathrm{Na}_{2} \mathrm{SO}_{4}$ aqueous solution was employed as the electrolyte $(\mathrm{pH}=7)$. The plot of alternating-current impedance (EIS) was obtained under a bias of $1.23 \mathrm{~V} v s$. RHE. The potential (vs. $\mathrm{Ag} / \mathrm{AgCl}$ ) was 
converted to the reversible hydrogen electrode (RHE) according to the Nernst equation: $E_{R H E}=E+0.05916 p H+E_{0}$, where $E_{R H E}$ is the potential $v s$. RHE, $E_{0}=0.1976 \mathrm{~V}$ at $25^{\circ} \mathrm{C}$, and $E$ is the measured potential vs. $\mathrm{Ag} / \mathrm{AgCl}$.

\subsection{Time-Resolved Diffuse Reflectance Spectral Measurements}

The femtosecond diffuse reflectance spectral measurement was performed with the pump and probe method using a regeneratively amplified titanium sapphire laser (Spectra-Physics, Spitfire Pro F, 1 kHz) pumped by a Nd:YLF laser (Spectra-Physics, Empower 15). The seed pulse was generated by a titanium sapphire laser (Spectra-Physics, Mai Tai VFSJW; fwhm 80 fs). The output (320 or $420 \mathrm{~nm}, 3 \mathrm{~mJ}$ pulse $^{-1}$ ) of the optical parametric amplifier (Spectra-Physics, OPA-800CF-1) was used as the excitation pulse. A white light continuum pulse, which was generated by focusing the residual of the fundamental light on a sapphire crystal after the computer controlled optical delay, was divided into two parts and used as the probe and the reference lights, of which the latter was used to compensate the laser fluctuation. Both probe and reference lights were directed to the sample powder coated on the glass substrate, and the reflected lights were detected by a linear InGaAs array detector equipped with the polychromator (Solar, MS3504). The pump pulse was chopped by the mechanical chopper synchronized to the laser repetition rate and overlapped with the probe pulse at the sample, resulting in a pair of spectra with and without the pump, from which the absorption change (\% Abs) induced by the pump pulse was estimated. All measurements were carried out at room temperature. 


\section{Results and discussion}

\subsection{Characterization of $g-C_{3} N_{4} / N L T O$}

The phase identification of the samples was performed by XRD. Figure 1A shows XRD patterns of the pristine LTO, NLTO, NLTO loaded with $10 \mathrm{wt} \% \mathrm{~g}-\mathrm{C}_{3} \mathrm{~N}_{4}$ ( $\left.\mathrm{g}-\mathrm{C}_{3} \mathrm{~N}_{4} / \mathrm{NLTO}\right)$, and LTO loaded with $10 \mathrm{wt} \% \mathrm{~g}-\mathrm{C}_{3} \mathrm{~N}_{4}\left(\mathrm{~g}-\mathrm{C}_{3} \mathrm{~N}_{4} / \mathrm{LTO}\right)$. All diffraction peaks of LTO and $g-\mathrm{C}_{3} \mathrm{~N}_{4} / \mathrm{LTO}$ are indexed to the monoclinic phase $\mathrm{La}_{2} \mathrm{Ti}_{2} \mathrm{O}_{7}$ with a perovskite structure belonging to the P21 space group (JCPDS No. 70-0903). After the hydrothermal retreatment of LTO in TEOA solution, $\mathrm{LaCO}_{3} \mathrm{OH}$ was formed accompanying with NLTO (Fig. S1a). To remove the byproduct, the obtained dark yellow precipitates were treated with diluted nitric acid. Subsequently, XRD patterns of the resultant bright yellow precipitates in Fig. 1A are assigned to pure LTO. The expansion of the crystal lattice resulted from the doping was clearly observed with the shift of the diffraction peaks toward the lower angle range, since the ion radius of $\mathrm{N}^{2-}$ $(1.29 \AA)$ is larger than that of $\mathrm{O}^{2-}(1.21 \AA)[25,26]$. For stacked $g-\mathrm{C}_{3} \mathrm{~N}_{4}$ nanosheets obtained by the thermal oxidation process, the reflection peak at $12.6^{\circ}$ in Fig. S1b corresponds to the c-plane of stacking $\mathrm{g}-\mathrm{C}_{3} \mathrm{~N}_{4}$ [27]. However, no signal assigned to g- $\mathrm{C}_{3} \mathrm{~N}_{4}$ was detectable in the composites even though the percentage reaches $15 \mathrm{wt} \%$ (Fig. S1c and d), possibly as a result of its low crystallinity. This can also be explained by the fact that $\mathrm{g}-\mathrm{C}_{3} \mathrm{~N}_{4}$ is ultrathin and is uniformly distributed on the LTO nanosheets, as shown in the TEM and AFM images below.

The compositions of the samples were analyzed by XPS, showing that NLTO 
contains 5.8 at\% of $\mathrm{N}$ after TEOA treatment, which is larger than that of NLTO obtained by annealing under $\mathrm{NH}_{3}$ flow [8]. The high chemical purity of the samples is demonstrated in Fig. S2, consisting solely of La, Ti, O, C and N. N 1s core-level XPS spectrum of NLTO in Fig. 1B-a displays two distinct peaks, N 1s peak (P1) at 400.2 $\mathrm{eV}$ assigned to the Ti-(N-O) bond $[8,28]$ and $\mathrm{N}$ 1s peak (P2) at $398.6 \mathrm{eV}$ assigned to the Ti-N bond, indicating that some $\mathrm{N}$ atoms are directly bonded to Ti atoms in the $\mathrm{TiO}_{6}$ octahedron $[28,29]$. $\mathrm{N}$ 1s spectrum of $\mathrm{g}-\mathrm{C}_{3} \mathrm{~N}_{4}$ (Fig. 1B-d) confirms the existence of $\mathrm{sp}^{2}$-hybridized $\mathrm{N}(\mathrm{P} 4)$ at $396.7 \mathrm{eV}$, the tertiary $\mathrm{N}\left(\mathrm{N}(\mathrm{C})_{3}, \mathrm{P} 3\right)$ at $398.2 \mathrm{eV}$, and the amino functional groups (P5) at $400.5 \mathrm{eV}$ [30]. N 1s peaks of the composites in Figs. 1B-b and c correspond to those of their compositions, as compared with Figs. 1B-a and $\mathrm{d}$. The assignment of $\mathrm{N} 1 \mathrm{~s}$ peaks was further confirmed by the difference in the $\mathrm{Ti}$ $2 p$ peaks with and without $\mathrm{N}$-doping. Both LTO and $\mathrm{g}-\mathrm{C}_{3} \mathrm{~N}_{4} / \mathrm{LTO}$ exhibit symmetric Ti 2p3/2 peaks at $458.0 \mathrm{eV}$ (Figs. S3A-a and d), which are assigned to Ti-O bond. In contrast, Ti $2 \mathrm{p} 3 / 2$ peaks of the NLTO are asymmetric and are deconvoluted into two components (Figs. S3A-b and c). The major component is assigned to the Ti-O bond, while the peak at $458.8 \mathrm{eV}$ is assigned to Ti-N bond [29]. In addition, the peaks of $\mathrm{Ti}$ 2p3/2 in NLTO shift to lower binding energy, indicating that the electron density around $\mathrm{Ti}$ in NLTO becomes higher. It can be attributed to the formation of $\mathrm{Ti}^{3+}$, which may cause oxygen vacancies $\left(V_{\mathrm{O}}\right)$ or interstitial Ti atoms $\left(\mathrm{Ti}_{\mathrm{i}}\right)$ [31,32]. All four samples, LTO, NLTO, g-C $3 \mathrm{~N}_{4} / \mathrm{NLTO}$, and $\mathrm{g}_{-} \mathrm{C}_{3} \mathrm{~N}_{4} / \mathrm{LTO}$, have typical O 1 s spectrum with two peaks respectively located at $532.5 \mathrm{eV}$ (adsorbed oxygen) and $531.0 \mathrm{eV}$ 
(Ti-O bonds), whereas for NLTO and $\mathrm{g}-\mathrm{C}_{3} \mathrm{~N}_{4} / \mathrm{NLTO}$, there is an additional peak at $528.6 \mathrm{eV}$ which corresponds to the Ti-(N-O) bond (Figs. S3B-b and c). There is no change in the La $3 \mathrm{~d}$ doublet peak before and after N-doping and hybridization of g- $\mathrm{C}_{3} \mathrm{~N}_{4}$ (Fig. S3C), suggesting that doped $\mathrm{N}$ atoms are preferentially positioned in the $\mathrm{TiO}_{6}$ octahedron slabs, in agreement with the previous studies $[8,33] . \mathrm{C} 1 \mathrm{~s}$ spectrum derived from $\mathrm{g}-\mathrm{C}_{3} \mathrm{~N}_{4}$ (Fig. S3D) is deconvoluted into three components, C-O, C-C, and $\mathrm{N}-\mathrm{C}=\mathrm{N}$ bands $[30,34,35]$.

The general morphologies of the materials were characterized by FESEM and TEM. Figure 2a shows a typical SEM image of LTO, and it can be clearly seen that pure LTO is composed of smooth and irregular nanosheets with sizes ranging from $100 \mathrm{~nm}$ to $600 \mathrm{~nm}$. N-doping does not change the sheet-like structure of LTO (Fig. $2 \mathrm{~b}$ ), which is attributed to the topochemical transformation between surface atoms of LTO crystals suspended in a liquid phase and TEOA molecular species in solution under hydrothermal condition [36-38]. The EDS analysis in Fig. S4 exhibits La, Ti, O, and $\mathrm{N}$ molar ratio of $0.72: 1: 1.74: 0.2$ in NLTO, consistent with the XPS analysis. The representative $\mathrm{g}-\mathrm{C}_{3} \mathrm{~N}_{4}$ nanosheets in Fig. 2c appear as loose and soft agglomerates with a size of tens of micrometers. Figures $2 \mathrm{~d}$ and e display the morphologies of $\mathrm{g}_{-} \mathrm{C}_{3} \mathrm{~N}_{4} / \mathrm{LTO}$ and $\mathrm{g}-\mathrm{C}_{3} \mathrm{~N}_{4} / \mathrm{NLTO}$, respectively. Clearly, LTO and NLTO nanosheets are irregularly dispersed on the surface of the $\mathrm{g}-\mathrm{C}_{3} \mathrm{~N}_{4}$ nanosheets. A solid construction can be achieved by such 2D/2D layered composites due to the large interface area between the sheets. Figure 3 displays TEM and HRTEM images of LTO, 
NLTO, g- $\mathrm{C}_{3} \mathrm{~N}_{4}$, and $\mathrm{g}-\mathrm{C}_{3} \mathrm{~N}_{4} / \mathrm{NLTO}$. Fringes with lattice spacing of $0.28 \mathrm{~nm}$ in LTO and NLTO were observed clearly (inset of Figs. 3a and b), which are in accordance with (012) plane of $\mathrm{La}_{2} \mathrm{Ti}_{2} \mathrm{O}_{7}$, implying a preserved crystal structure of LTO after $\mathrm{N}$-doping. The appearance of a basic sheet of $\mathrm{g}-\mathrm{C}_{3} \mathrm{~N}_{4}$ in Fig. $3 \mathrm{c}$ is similar to that of graphite. The inserted HRTEM image demonstrates the rough surface and amorphous characterization of the $\mathrm{g}-\mathrm{C}_{3} \mathrm{~N}_{4}$ sheets. From the typical TEM image of $\mathrm{g}-\mathrm{C}_{3} \mathrm{~N}_{4} / \mathrm{NLTO}$ nanocomposites in Fig. 3d, it can be seen that NLTO nanosheets flatly lie on the surface of $2 \mathrm{D} \mathrm{g}-\mathrm{C}_{3} \mathrm{~N}_{4}$, displaying large interfacial areas because of the $2 \mathrm{D} / 2 \mathrm{D}$ interface between $\mathrm{g}-\mathrm{C}_{3} \mathrm{~N}_{4}$ and NLTO. Moreover, by AFM measurement, the thickness of $\mathrm{g}-\mathrm{C}_{3} \mathrm{~N}_{4}$ and NLTO are determined to be $\sim 2 \mathrm{~nm}$ and $7 \mathrm{~nm}$, respectively (Fig. S5). As the nanomaterials are thinner, the transport distance of charge carriers from photoexcited sites to reaction surface or interface becomes shorter [39]. The interfacial area in $\mathrm{g}-\mathrm{C}_{3} \mathrm{~N}_{4} / \mathrm{NLTO}$ composite system was also estimated. In Fig S6, $\sim 83 \%$ of $\mathrm{g}-\mathrm{C}_{3} \mathrm{~N}_{4}$ nanosheets are covered by NLTO nanosheets, exhibiting a large interfacial area of $0.83 \mu \mathrm{m}^{2}$ per $\mu \mathrm{m}^{2} \mathrm{~g}-\mathrm{C}_{3} \mathrm{~N}_{4}$. Hence, it is expected that the large interfacial area provides sufficient interface for efficient charge transfer, and an ultrathin heterostructure is beneficial to shorten the charge transport distance [40].

\subsection{Band alignment of $g-C_{3} N_{4} / N L T O$}

The optical responses of LTO were variable with the $\mathrm{N}$ doping and hybridization of $\mathrm{g}_{-} \mathrm{C}_{3} \mathrm{~N}_{4}$, as shown in the UV-vis diffuse reflectance spectra (DRS) in Fig. 4a. Compared to pristine LTO, the absorption edges of N-doped samples (NLTO and 
g- $\mathrm{C}_{3} \mathrm{~N}_{4} / \mathrm{NLTO}$ ) show a red shift from 371 to $391 \mathrm{~nm}$. Accordingly, the derived electronic band gaps from the Tauc plots are $3.17 \mathrm{eV}$ for NLTO and $3.34 \mathrm{eV}$ for LTO nanosheets (inset in Fig. 4a). Remarkably, add-on shoulders are imposed from $380 \mathrm{~nm}$ to $550 \mathrm{~nm}$ due to $\mathrm{N}$ doping with the mixed $\mathrm{N} 2 \mathrm{p}, \mathrm{O} 2 \mathrm{p}$ and $\mathrm{Ti} 3 \mathrm{~d}$ states in the midgap, as discussed below. $\mathrm{g}-\mathrm{C}_{3} \mathrm{~N}_{4}$ nanosheets exhibit the visible light response with absorption edges at $477 \mathrm{~nm}$, corresponding to $2.65 \mathrm{eV}$ of electronic band gap. g- $\mathrm{C}_{3} \mathrm{~N}_{4} / \mathrm{NLTO}$ composite displays higher visible light harvesting ability than NLTO and g- $\mathrm{C}_{3} \mathrm{~N}_{4} / \mathrm{LTO}$ due to $\mathrm{N}$-doping and g- $\mathrm{C}_{3} \mathrm{~N}_{4}$ modification.

Mott-Schottky measurements were analyzed to elucidate the flat-band potential $V_{\mathrm{fb}}$, which are determined to be $-0.72,-0.63$, and $-0.83 \mathrm{~V} v s$ RHE for undoped LTO, NLTO, and g- $\mathrm{C}_{3} \mathrm{~N}_{4}$, respectively (Fig. 4b). The result is consistent with a type II band alignment between LTO and $\mathrm{g}-\mathrm{C}_{3} \mathrm{~N}_{4}$, since the gap between $V_{\mathrm{fb}}$ and bottom edge of the conduction band $(\mathrm{CB})$ is assumed to be small $(\sim 0.2 \mathrm{eV})$ and similar for LTO and g- $\mathrm{C}_{3} \mathrm{~N}_{4}$ [41]. In type II heterostructure band alignment, the position of $\mathrm{CB}$ and valence band (VB) of $\mathrm{g}-\mathrm{C}_{3} \mathrm{~N}_{4}$ is both higher than that of LTO. The band bending at the interface of type II heterojunction due to the difference of chemical potential between g- $\mathrm{C}_{3} \mathrm{~N}_{4}$ and LTO induces a built-in field, which promotes the photoexcited electrons to facilely move from $\mathrm{CB}$ of $\mathrm{g}-\mathrm{C}_{3} \mathrm{~N}_{4}$ to LTO, and holes move from VB of LTO to g- $\mathrm{C}_{3} \mathrm{~N}_{4}[42,43]$. CB of the LTO nanosheets declined $0.09 \mathrm{eV}$ after N-doping. Considering the $0.17 \mathrm{eV}$ decrease in the bandgap and a positive shift of $0.09 \mathrm{eV}$ in $\mathrm{CB}$, VB of NLTO is calculated to be upshifted by $0.08 \mathrm{eV}$. We have investigated the 
influence of $\mathrm{N}$-doping on the crystal and electronic structures of $\mathrm{La}_{2} \mathrm{Ti}_{2} \mathrm{O}_{7}$ using first principles calculations [11]. Localized mid-gap states formed when $\mathrm{N}$ substitutions for $\mathrm{O}\left(\mathrm{N}_{\mathrm{s}}\right)$ co-exist with interstitial $\mathrm{Ti}$ atoms $\left(\mathrm{Ti}_{\mathrm{i}}\right)$, due to the existence of $\mathrm{Ti}^{3+}$ (see XPS analysis). Two localized energy bands, composed of mainly Ti $3 d$, and the least $\mathrm{O} 2 \mathrm{p}$ states, covered a wide range below the conduction band maximum (CBM). Another mixed $\mathrm{N} 2 \mathrm{p}, \mathrm{O} 2 \mathrm{p}$ and $\mathrm{Ti} 3 \mathrm{~d}$ localized energy band was located $0.4 \mathrm{~V}$ above the valence band minimum (VBM) according to the value calculated from the density functional theory (DFT). To sum up, besides narrowing the band gap, $\mathrm{N}$ doping resulted in localized energy bands that cause the add-on shoulder absorption.

\subsection{Photocatalytic activity of $g-C_{3} N_{4} / N L T O$}

The photocatalytic activities of $\mathrm{g}-\mathrm{C}_{3} \mathrm{~N}_{4} / \mathrm{NLTO}$ in oxidative degradation of $\mathrm{MO}$ and reductive generation of $\mathrm{H}_{2}$ were examined. Figure 5a shows the degradation of $\mathrm{MO}$ in the presence of LTO, NLTO, g- $\mathrm{C}_{3} \mathrm{~N}_{4} / \mathrm{LTO}$, and $\mathrm{g}-\mathrm{C}_{3} \mathrm{~N}_{4} / \mathrm{NLTO}$ with equivalent g- $\mathrm{C}_{3} \mathrm{~N}_{4}$ loadings (10 wt $\%$ ), where $C_{\mathrm{o}}$ and $C$ are the concentrations of $\mathrm{MO}$ before and after the visible light irradiation, respectively. The decomposition of MO in the absence of photocatalyst or light was less than 5\%, which was within the experimental tolerance range. As compared to LTO with feeble response to visible light, NLTO and/or g- $\mathrm{C}_{3} \mathrm{~N}_{4} /$ LTO showed markedly improvement of the oxidative efficiency. It is clearly shown that $\mathrm{g}-\mathrm{C}_{3} \mathrm{~N}_{4} / \mathrm{NLTO}$ with optimal ratio of $\mathrm{g}-\mathrm{C}_{3} \mathrm{~N}_{4}$ to NLTO exhibit the highest photocatalytic activity due to the strongest ability of the visible light harvesting and efficient charge separation. Subsequently, a promising and synergetic 
improvement in the photodegradation activity was observed on $\mathrm{g}_{-} \mathrm{C}_{3} \mathrm{~N}_{4} / \mathrm{NLTO}$, with $\mathrm{N}$-doping and hybridization of $\mathrm{g}-\mathrm{C}_{3} \mathrm{~N}_{4}$ being found to play crucial role in the degradation activity (Fig. S7). NLTO nanosheets show high photocatalytic activity under the visible and UV light irradiation. With the increase in the loading amounts of g- $\mathrm{C}_{3} \mathrm{~N}_{4}$ less than $10 \mathrm{wt} \%$, the degradation efficiency gradually increases with an increase of the number of photons absorbed by $\mathrm{g}-\mathrm{C}_{3} \mathrm{~N}_{4}$ nanosheets (Fig. S7b). The highest efficiency was obtained at an optimal value of $10 \mathrm{wt} \%$, where approximately $44 \%$ and $95 \%$ of MO was removed under the visible and UV light irradiation within 3 $\mathrm{h}$ and $1 \mathrm{~h}$, respectively. Similar results were observed for $\mathrm{g}-\mathrm{C}_{3} \mathrm{~N}_{4} / \mathrm{LTO}$, except that 7.5 wt\% was found to be the optimal ratio in the case of visible light irradiation (Fig. S7a).

We measured the amounts of $\mathrm{H}_{2}$ evolved using the composites loaded with Pt as co-catalysts in aqueous solutions containing methanol as a sacrificial electron donor during the visible and simulative solar light irradiation. As shown in Fig. S8, Pt nanoparticles with size of $\sim 2 \mathrm{~nm}$ uniformly distribute on the surface of the composite system. Under solar light irradiation (Fig. S9), N-doping significantly enhances the photocatalytic $\mathrm{H}_{2}$ evolution rate of LTO nanosheets from 0.04 to $0.20 \mathrm{mmol} \mathrm{h}^{-1} \mathrm{~g}^{-1}$. The optimal $\mathrm{g}-\mathrm{C}_{3} \mathrm{~N}_{4} / \mathrm{NLTO}$ with $10 \mathrm{wt} \% \mathrm{~g}-\mathrm{C}_{3} \mathrm{~N}_{4}$ gives the $\mathrm{H}_{2}$ evolution rate of 0.43 mmol $\mathrm{h}^{-1} \mathrm{~g}^{-1}$, which is 10 and 2 times larger than those of LTO and NLTO, respectively. The contribution of $\mathrm{N}$-doping and $\mathrm{g}-\mathrm{C}_{3} \mathrm{~N}_{4}$ modification in visible light induced activity is further examined by using a $\lambda>400 \mathrm{~nm}$ cutoff filter (Fig. 5b). The 
photocatalytic activity of LTO was negligibly low, while $\mathrm{H}_{2}$ generation by NLTO, g- $\mathrm{C}_{3} \mathrm{~N}_{4} / \mathrm{LTO}$, and $\mathrm{g}-\mathrm{C}_{3} \mathrm{~N}_{4} / \mathrm{NLTO}$ was observed, confirming the visible light irradiation due to $\mathrm{N}$ dopants and $\mathrm{g}-\mathrm{C}_{3} \mathrm{~N}_{4}$ loading. Furthermore, the apparent quantum efficiency (AQE) at each centered wavelength of the monochromatic light was measured. The trends of $\mathrm{H}_{2}$ evolution rates by the photocatalysts (LTO, NLTO, and $\mathrm{g}-\mathrm{C}_{3} \mathrm{~N}_{4} / \mathrm{NLTO}$ ) are generally consistent with their UV/Vis DRS (Fig. 5c), indicating the photocatalytic activities are driven by the absorbed light. The photocatalytic $\mathrm{H}_{2}$ evolution rates by the composite are always much higher than those by NLTO under the same irradiation. Under the 400-nm monochromatic light irradiation, the AQE of g- $\mathrm{C}_{3} \mathrm{~N}_{4} / \mathrm{NLTO}(2.1 \%)$ is 4 times higher than that of NLTO photocatalyst (0.5\%). An efficient charge transfer between $\mathrm{g}-\mathrm{C}_{3} \mathrm{~N}_{4}$ and NLTO exists in the photocatalyst, which is confirmed by the femtosecond time-resolved diffuse reflection spectral measurement as described below.

\subsection{Charge transfer on $g-C_{3} N_{4} / N L T O$}

To clarify the fast interfacial charge transfer in $g-\mathrm{C}_{3} \mathrm{~N}_{4} / \mathrm{NLTO}$, we performed the EIS measurement and femtosecond TDR spectroscopy. The diameters of the arc radius on the EIS Nynquist plot of NLTO and/or g- $_{3} \mathrm{~N}_{4} / \mathrm{NLTO}$ electrodes are smaller than that of LTO electrode (Fig. S10). The smaller the arc radius of an EIS Nynquist plot, the higher the charge separation [44]. Thus, with regard to NLTO, the electron-hole pairs are easily separated and transferred to the nanosheet surface which is due to the alteration of electronic band structure. The addition of $\mathrm{g}-\mathrm{C}_{3} \mathrm{~N}_{4}$ introduces an interfacial 
electron transfer from $\mathrm{g}-\mathrm{C}_{3} \mathrm{~N}_{4}$ to NLTO due to the type II band alignment.

As stated above, we have recently systematically studied the interactions between native donors and $\mathrm{N}_{\mathrm{s}}$ in electronic band structure of NLTO [11]. Computational results suggested that when a $\mathrm{Ti}$ atom at an interstitial site $\left(\mathrm{Ti}_{\mathrm{i}}\right)$ was bound with $\mathrm{N}_{\mathrm{s}}$, dispersedly distributed localized energy states formed just above the VBM and below the CBM. The suggested mechanism associated with the results of femtosecond TDR spectroscopic measurement in Fig. 6a gives a reasonable explanation for the high photocatalytic activity of NLTO under the visible and UV light irradiation. Upon the visible light irradiation of NLTO at $420 \mathrm{~nm}$, electrons are excited from mixed $\mathrm{N} 2 \mathrm{p} / \mathrm{O} 2 \mathrm{p} / \mathrm{Ti} 3 \mathrm{~d}$ levels to the CB Ti $3 \mathrm{~d} / \mathrm{O} 2 \mathrm{p}$ to generate charge carriers (hole-electron pairs) having the broad transient absorption around $900 \mathrm{~nm}$. The transient absorption decayed with three lifetimes of a few to $1000 \mathrm{ps}\left(\tau_{1}, \tau_{2}\right.$, and $\tau_{3}$, Table 1$) . \tau_{1}=2.9 \mathrm{ps}$ for NLTO are greatly influenced by additional density of states (DOS) above the VB that increases relaxation of energetic carriers to the shifted band edge [45]. After charges are trapped in the localized trapping sites in the lifetime of $\tau_{2}$ $=38 \mathrm{ps}$, the charge recombination occurs in the lifetime of $\tau_{3}=864 \mathrm{ps}$ in NLTO. The hybridized localized states in NLTO (Fig. 6b) are dispersedly distributed in a large energy range, even more disperse than the valence band top and conduction band bottom energy levels [11]. The charge carriers in the de-localized energy states can transport freely, unlike in the common localized energy state [46]. As a result, nitrogen doping enables the efficient visible-light-driven photocatalytic activity of 
LTO. The hybridization of NLTO with $\mathrm{g}_{-} \mathrm{C}_{3} \mathrm{~N}_{4}$ further enhances the photocatalytic degradation and $\mathrm{H}_{2}$ production performance through generating a type II heterostructure at the maximum facet-to-facet $2 \mathrm{D}$ connection. The longer lifetimes of $\tau_{1}=5.6 \mathrm{ps}$ and $\tau_{2}=68 \mathrm{ps}$ were observed for $\mathrm{g}-\mathrm{C}_{3} \mathrm{~N}_{4} / \mathrm{NLTO}$ compared with those for NLTO, showing the similar photocatalytic activity under the UV irradiation (see below). On the other hand, the shorter lifetime of $\tau_{3}=496 \mathrm{ps}$ was detected for g- $\mathrm{C}_{3} \mathrm{~N}_{4} / \mathrm{NLTO}$ than for NLTO, indicating the fast charge transfer from CB electrons of $\mathrm{g}-\mathrm{C}_{3} \mathrm{~N}_{4}$ to $\mathrm{CB}$ of NLTO [39]. The time profile of the transient adsorption at $900 \mathrm{~nm}$ for pristine LTO under visible laser irradiation is not discussed here, because it is probably resulted from unknown impurities [36]. Under the UV laser irradiation at $320 \mathrm{~nm}$, LTO showed three decay profiles with lifetimes of $5.1 \mathrm{ps}\left(\tau_{1}\right), 55 \mathrm{ps}\left(\tau_{2}\right)$, and 463 ps $\left(\tau_{3}\right)$ (Fig. 6a and Table 1). The generated electrons and holes on pristine LTO are usually trapped at the surface defects $\left(\tau_{1}\right)$ separately to react with water or organic molecules with substantial photocatalytic efficiency. Compared with those for LTO, decay lifetimes of $9.3 \mathrm{ps}\left(\tau_{1}\right), 74 \mathrm{ps}\left(\tau_{2}\right)$, and $495 \mathrm{ps}\left(\tau_{3}\right)$ were observed to show the slower charge recombination process for NLTO, due to the decreased electron-hole recombination on $\mathrm{N} 2 \mathrm{p} / \mathrm{O} 2 \mathrm{p} / \mathrm{Ti} 2 \mathrm{p}$ trap state. Thus, the efficient charge separation of NLTO was observed with the higher photocatalytic activity under the UV irradiation compared to that for LTO. $\mathrm{g}-\mathrm{C}_{3} \mathrm{~N}_{4} / \mathrm{NLTO}$ exhibited high photocatalytic activity with the longer lifetimes of charge carriers, $6.8 \mathrm{ps}\left(\tau_{1}\right), 96 \mathrm{ps}\left(\tau_{2}\right)$, and $627 \mathrm{ps}\left(\tau_{3}\right)$, under the same irradiation condition. The band bending at the interface of type II heterojunction 
due to the difference of potential energies between NLTO and $\mathrm{g}-\mathrm{C}_{3} \mathrm{~N}_{4}$ induces a built-in field (Fig. 6b), which promotes the electron-hole pair to facilely migrate to the opposite directions [41]. The large interfacial area of $2 \mathrm{D}$ layered $\mathrm{g}-\mathrm{C}_{3} \mathrm{~N}_{4} / \mathrm{NLTO}$ composite results in sufficient interface for efficient charge transfer to generate an excellent type II heterojunction. The ultrathin g- $\mathrm{C}_{3} \mathrm{~N}_{4} / \mathrm{NLTO}$ heterostructure $(\sim 2 \mathrm{~nm}$ thick $\mathrm{g}-\mathrm{C}_{3} \mathrm{~N}_{4}$ and $7 \mathrm{~nm}$ thick NLTO) shortens the charge transport time and distance, decreasing the recombination of photogenerated electron-hole pairs during migration. Both of them are favorable to longer lifetime of charge carriers.

\section{Conclusions}

Approximately $7 \mathrm{~nm}$ thick NLTO nanosheets hybridized with $2 \mathrm{~nm}$ thick g- $\mathrm{C}_{3} \mathrm{~N}_{4}$ layered composites, g- $\mathrm{C}_{3} \mathrm{~N}_{4} / \mathrm{NLTO}$, have been synthesized from a simple two-step hydrothermal process followed by a modified thermal oxidation etching method. g- $\mathrm{C}_{3} \mathrm{~N}_{4} / \mathrm{NLTO}$ was characterized by a variety of measurements and the photocatalytic activities were evaluated from $\mathrm{H}_{2}$ production from water splitting and $\mathrm{MO}$ decomposition in aqueous solution. It was found that NLTO nanosheets form the interface with the surface of $2 \mathrm{D} \mathrm{g}-\mathrm{C}_{3} \mathrm{~N}_{4}$, with the maximum interface. $g-\mathrm{C}_{3} \mathrm{~N}_{4} / \mathrm{NLTO}$ exhibited a high AQE of $2.1 \%$ for photocatalytic $\mathrm{H}_{2}$ evolution under the 400-nm monochromatic light irradiation. Such high photocatalytic activities are explained by the properties of $\mathrm{g}-\mathrm{C}_{3} \mathrm{~N}_{4}$ and NLTO, efficient light absorption and electron transport ability. Electrochemical and spectroscopic measurements were performed to clarify the type II band alignment for favorable electron transfer from $\mathrm{g}-\mathrm{C}_{3} \mathrm{~N}_{4}$ to NLTO. The 
longer charge carrier lifetime of $\mathrm{g}_{-} \mathrm{C}_{3} \mathrm{~N}_{4} / \mathrm{NLTO}$ compared with LTO and NLTO measured by femtosecond time-resolved diffused reflectance (TDR) spectroscopy clearly indicates the high photocatalytic activity of $\mathrm{g}-\mathrm{C}_{3} \mathrm{~N}_{4} / \mathrm{NLTO}$ under the visible and UV laser irradiation. It is suggested that the large interface between 2D layered g- $\mathrm{C}_{3} \mathrm{~N}_{4}$ and NLTO as well as their ultrathin heterostructure lead to efficient interfacial charge transfer to generate the charge separation with longer lifetime.

\section{Acknowledgements}

This project is financially supported by the National Science Foundation of China under Grant No. 51472013, Beijing Key Discipline Foundation of Condensed Matter Physics, and China Scholarship Council (CSC). We are thankful for the help of the Comprehensive Analysis Center of SANKEN, Osaka University. This work has been partly supported by a Grant-in-Aid for Scientific Research (Project 25220806, 25288035, and others) from the Ministry of Education, Culture, Sports, Science and Technology (MEXT) of the Japanese Government.

\section{References}

[1] Z. Bian, T. Tachikawa, P. Zhang, M. Fujitsuka, T. Majima, J. Am. Chem. Soc. 136 (2014) 458-465.

[2] H.G. Kim, D.W. Hwang, J. Kim, Y.G. Kim, J.S. Lee, Chem. Commun. (1999) 1077-1078. 
[3] X.C. Wang, K. Maeda, A. Thomas, K. Takanabe, G. Xin, J.M. Carlsson, K. Domen, M. Antonietti, Nat. Mater. 8 (2009) 76-80.

[4] Y. Miseki, H. Kato, A. Kudo, Energy Environ. Sci. 2 (2009) 306-314.

[5] H.G. Yang, G. Liu, S.Z. Qiao, C.H. Sun, Y.G. Jin, S.C. Smith, J. Zou, H.M. Cheng, G.Q. Lu, J. Am. Chem. Soc. 131 (2009) 4078-4083.

[6] J. Jiang, K. Zhao, X.Y. Xiao, L.Z. Zhang, J. Am. Chem. Soc. 134 (2012) 4473-4476.

[7] H. Liu, M. Luo, J.C. Hu, T.F. Zhou, R. Chen, J.L. Li, Appl. Catal. B 140 (2013) $141-150$.

[8] F.K. Meng, Z.L. Hong, J. Arndt, M. Li, M.J. Zhi, F. Yang, N.Q. Wu, Nano Res. 5 (2012) 213-221.

[9] S. Hu, L. Jia, B. Chi, J. Pu, L.J. Jian, J. Power Sources 266 (2014) 304-312.

[10] S. Hu, M. Zhu, ChemPlusChem 81 (2016) 1-8.

[11] J. Zhang, W. Dang, Z. Ao, S. K. Cushing, N. Wu, Phys. Chem. Chem. Phys. 17 (2015) 8994-9000.

[12] F. Meng, S.K. Cushing, J. Li, S. Hao, N. Wu, ACS Catal. 5 (2015) 1949-1955.

[13] F Meng, J. Li, Z. Hong, M. Zhi, A. Sakla, C. Xiang, N. Wu, Catal. Today 199 (2013) 48-52.

[14] R. Asahi, T. Morikawa, T. Ohwaki, K. Aoki, Y. Taga, Science 293 (2001) 269-271.

[15] C. Burda, Y.B. Luo, X.B. Chen, A.C.S. Samia, J. Stout, J.L. Gole, Nano Lett. 3 (2003) 1049-1051. 
[16] T. Tachikawa, Y. Takai, S. Tojo, M. Fujitsuka, H. Irie, K. Hashimoto, T. Majima, J. Phys. Chem. B 110 (2006) 13158-13165.

[17] H. Irie, Y. Watanabe, K. Hashimoto, J. Phys. Chem. B 107 (2003) 5483-5486.

[18] Y. Li, H. Xu, S. Ouyang, D. Lu, X. Wang, D. Wang, J. Ye, J. Mater. Chem. A 4 (2016) 2943-2950.

[19] H. Yu, L. Shang, T. Bian, R. Shi, G.I.N. Waterhouse, Y. Zhao, C. Zhou, L.Z. Wu, C.H. Tung, T. Zhang, Adv. Mater. 28 (2016) 5080-5086.

[20] P. Niu, L. Zhang, G. Liu, H.M. Cheng, Adv. Funct. Mater. 22 (2012) 4763-4770.

[21] J. Low, S. Cao, J. Yu, S. Wageh, Chem. Commun. 50 (2014) 1076-10777.

[22] Y. Hou, Z. Wen, S. Cui, X. Feng, J. Chen, Nano Lett. 16 (2016) 2268-2277.

[23] Q. Xiang, J. Yu, M. Jaroniec, J. Am. Chem. Soc. 134 (2012) 6575-6578.

[24] F. Meng, J. Li, S.K. Cushing, M. Zhi, N. Wu, J. Am. Chem. Soc. 135 (2013) 10286-10289.

[25] I. Marozaua, A. Shkabkob, M. Döbelic, T. Lipperta, M. Mallepellc, C.W. Schneidera, A. Weidenkaffb, A. Wokaun, Acta Mater. 59 (2011) 7145-7154

[26] X. Cai, D. Hu, S. Deng, B. Han, Y. Wang, J. Wu, Y. Wang, Sensor Actuat. B 198 (2014) 402-410

[27] Q. Tay, P. Kanhere, C.F. Ng, S. Chen, S. Chakraborty, A.C.H. Huan, T.C. Sum, R. Ahuja, Z. Chen, Chem. Mater. 27 (2015) 4930-4933.

[28] A. Fujishima, X.T. Zhang, D.A. Tryk, Surf. Sci. Rep. 63 (2008) 515-582.

[29] J.F. Moulder, W.F. Stickle, P.E. Sobol, K.D. Bomben, Physical Electronics: Eden 
Prairie (1995).

[30] Q. Han, B. Wang, J. Gao, Z. Cheng, Y. Zhao, Z. Zhang, L. Qu, ACS Nano 10 (2016) 2745-2751.

[31] H. Liu, F. Shen, M. Xing, J. Zhang, M. Anpo, Catal. Lett. 144 (2014) 1494-1498.

[32] M, Xing, J, Zhang, F. Chen, B. Tian, Chem. Commun. 47 (2011) 4947-4949.

[33] A. Kasahara, K. Nukumizu, T. Takata, J.N. Kondo, M. Hara, H. Kobayashi, K. Domen, J. Phys. Chem. B 107 (2003) 791-797.

[34] Y. Kang, Y. Yang, L. Yin, X. Kang, G. Liu, H.M. Cheng, Adv. Mater. 27 (2015) $4572-4577$.

[35] Q. Han, C.G. Hu, F. Zhao, Z.P. Zhang, N. Chen, L.T. Qu, J. Mater. Chem. A 3 (2015) 4612-4619.

[36] P. Zhang, M. Fujitsuka, T. Majima, Appl. Catal. B 185 (2016) 181-188.

[37] V. Kalyani, B.S. Vasile, A. Ianculescu, M.T. Buscaglia, V. Buscaglia, P. Nanni, Cryst. Growth Des. 12 (2012), 4450-4456.

[38] F. Peng, L. Cai, L. Huang, H. Yu, H. Wang, J. Phys. Chem. Solids 69 (2008) $1657-1664$.

[39] S. Ida, T. Ishihara, J. Phys. Chem. Lett. 5 (2014) 2533-2542.

[40] Y. Ao, K. Wang, P. Wang, C. Wang, J. Hou, RSC Adv. 6 (2016) 48599-48609.

[41] J. Resasco, H. Zhang, N. Kornienko, N. Becknell, H. Lee, J. Guo, A.L. Briseno, P. Yang, ACS Cent. Sci. 2 (2016) 80-88.

[42] H. Li, Y. Zhou, W. Tu, J. Ye, Z. Zou, Adv. Funct. Mater. 25 (2015) 998-1013. 
[43] Y. Lu, D. Chu, M. Zhu, Y. Dua, P. Yang, Phys. Chem. Chem. Phys. 17 (2015) 17355-17361.

[44] C. Zhai, M. Zhu, F. Pang, D. Bin, C. Lu, M.C. Goh, P. Yang, Y. Du, ACS Appl. Mater. Interfaces 8 (2016) 5972-5980.

[45] B.T. Yost, S.K. Cushing, F. Meng, J. Bright, D.A. Bas, N. Wu, A.D. Bristow, Phys. Chem. Chem. Phys. 17 (2015) 31039-31043.

[46] N. Umezawa, J. Ye, Phys. Chem. Chem. Phys. 14 (2012) 5924-5934. 


\section{Captions of Figures}

Fig. 1 X-ray diffraction patterns (A) and high-resolution XPS spectra of N 1s (B) of LTO (a), NLTO (b), g- $\mathrm{C}_{3} \mathrm{~N}_{4} / \mathrm{NLTO}\left(10 \mathrm{wt} \% \mathrm{~g}-\mathrm{C}_{3} \mathrm{~N}_{4}\right)$ (c), and g- $\mathrm{C}_{3} \mathrm{~N}_{4} / \mathrm{LTO}$ (10 $\left.w t \% g-\mathrm{C}_{3} \mathrm{~N}_{4}\right)(\mathrm{d})$.

Fig. 2 SEM images of LTO (a), NLTO (b), g- $\mathrm{C}_{3} \mathrm{~N}_{4}$ (c), g- $\mathrm{C}_{3} \mathrm{~N}_{4} / \mathrm{NLTO}$ (d), and $\mathrm{g}-\mathrm{C}_{3} \mathrm{~N}_{4} / \mathrm{LTO}(\mathrm{e})$.

Fig. 3 TEM images of LTO (a), NLTO (b), g- $\mathrm{C}_{3} \mathrm{~N}_{4}$ (c), and $\mathrm{g}-\mathrm{C}_{3} \mathrm{~N}_{4} / \mathrm{NLTO}$ (d). Insets for HRTEM images.

Fig. 4 (a) UV-vis diffuse reflectance spectroscopy of different samples. Inset for the optical band gap energy estimation of LTO, NLTO, and $\mathrm{g}_{-} \mathrm{C}_{3} \mathrm{~N}_{4}$; (b) Mott-Schottky (M-S) plots of LTO, NLTO, and g- $\mathrm{C}_{3} \mathrm{~N}_{4}$.

Fig. 5 Photocatalytic degradation of $\mathrm{MO}$ (a) and $\mathrm{H}_{2}$ evolution (b) over different samples under the visible light irradiation. The error bars obtained through three times of repeated tests; (c) Steady-state diffuse reflectance spectra (left axis, solid lines) and AQE (right axis, symbols with dashed lines) of LTO, NLTO, and $g-\mathrm{C}_{3} \mathrm{~N}_{4} / \mathrm{NLTO}$.

Fig. 6 (a) Normalized transient absorption trace from LTO, NLTO, and g- $\mathrm{C}_{3} \mathrm{~N}_{4} / \mathrm{NLTO}$ after 420-nm or 320-nm laser irradiation; (b) Schematic illustration of the energetic distribution of electrons and holes in $\mathrm{g}-\mathrm{C}_{3} \mathrm{~N}_{4} / \mathrm{NLTO}$ under the visible (yellow arrow) and UV (purple arrow) irradiation. 

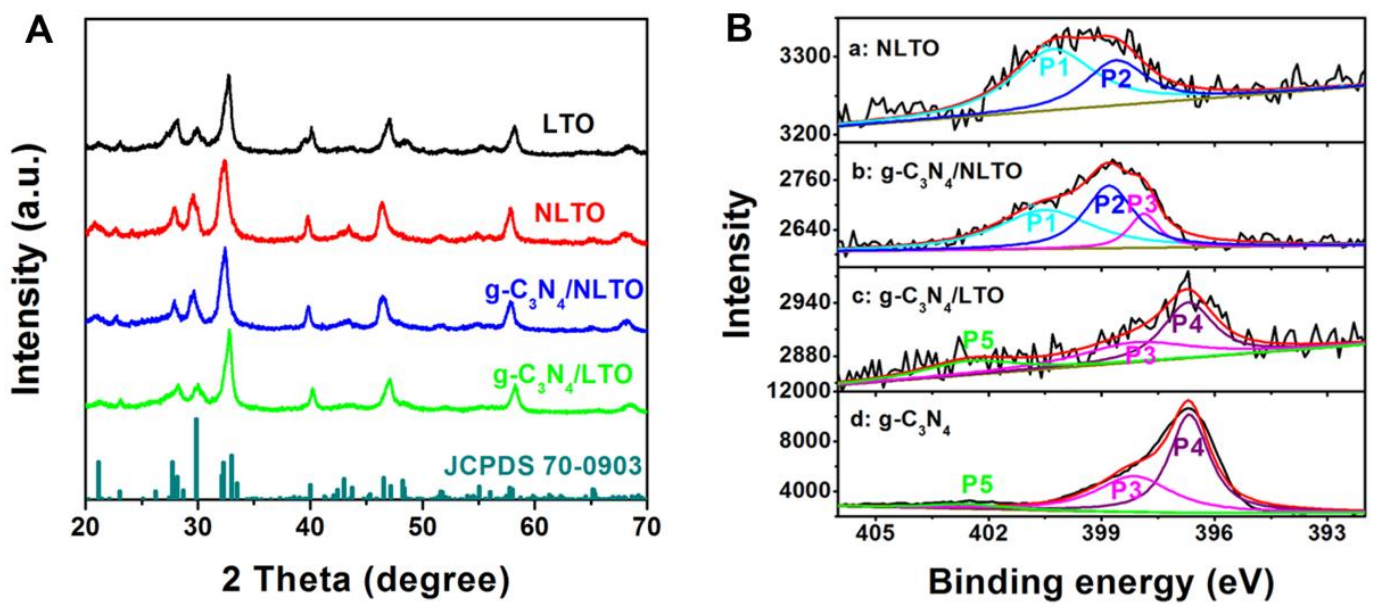

Fig. 1 

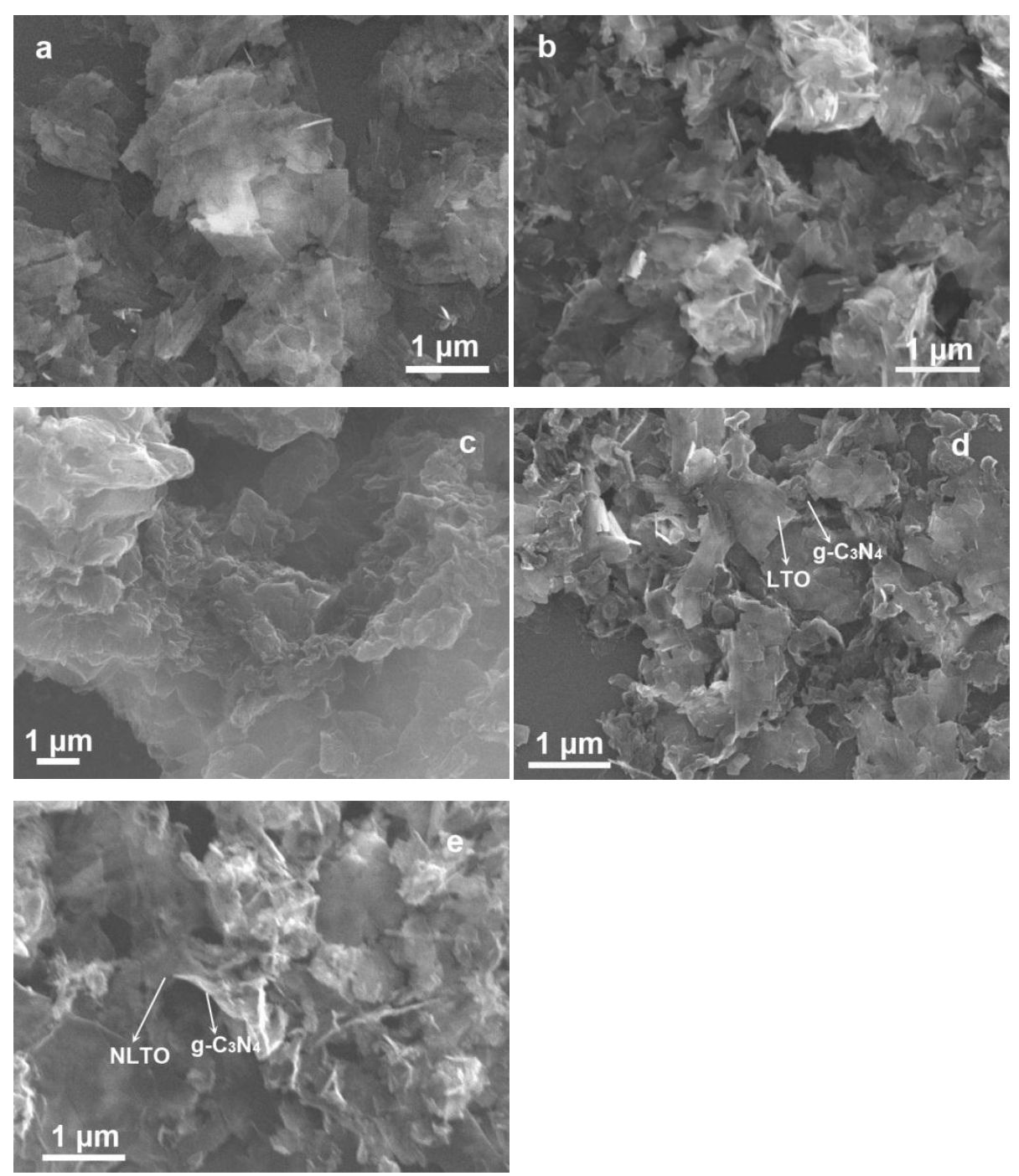

Fig. 2 

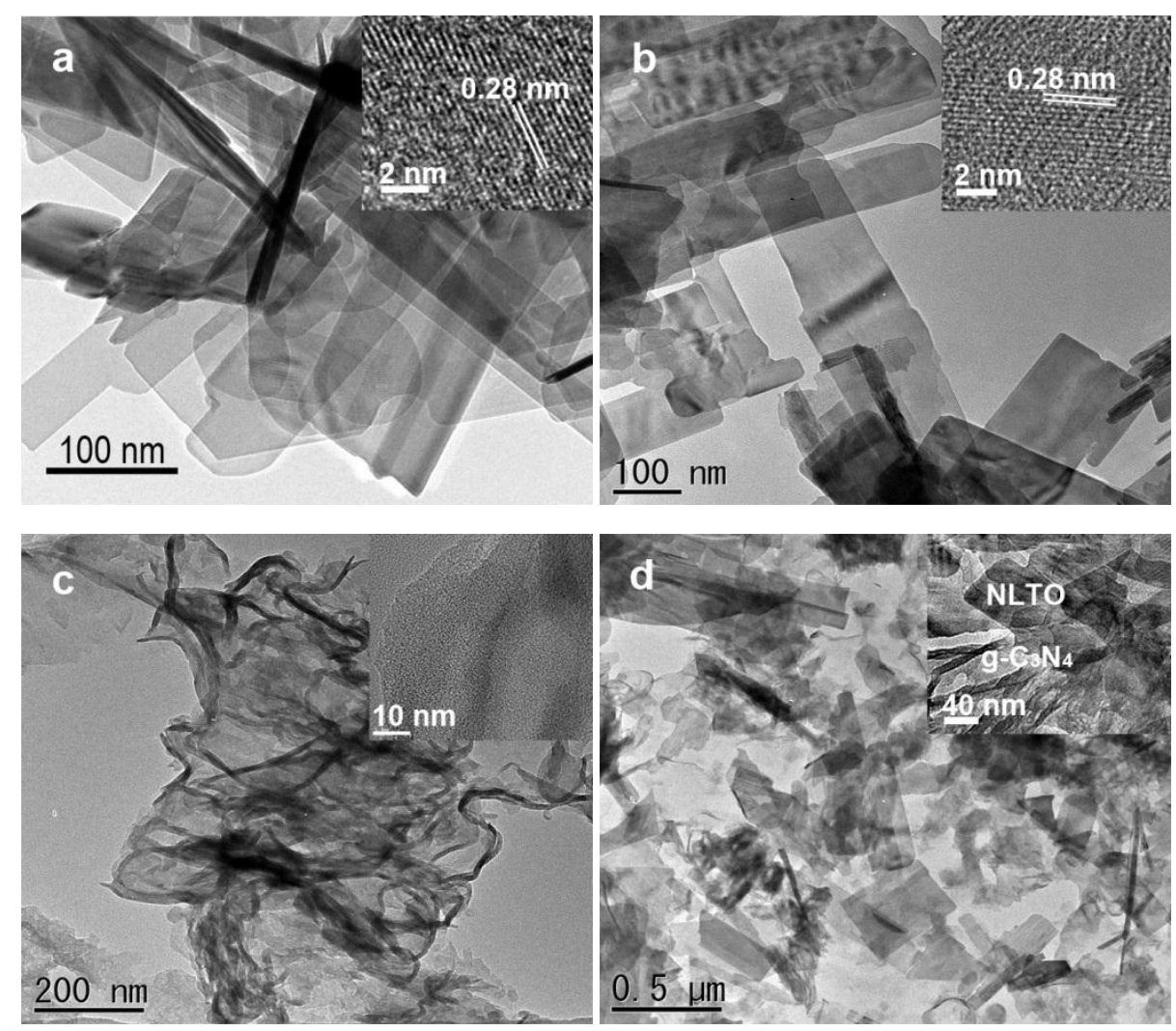

Fig. 3 

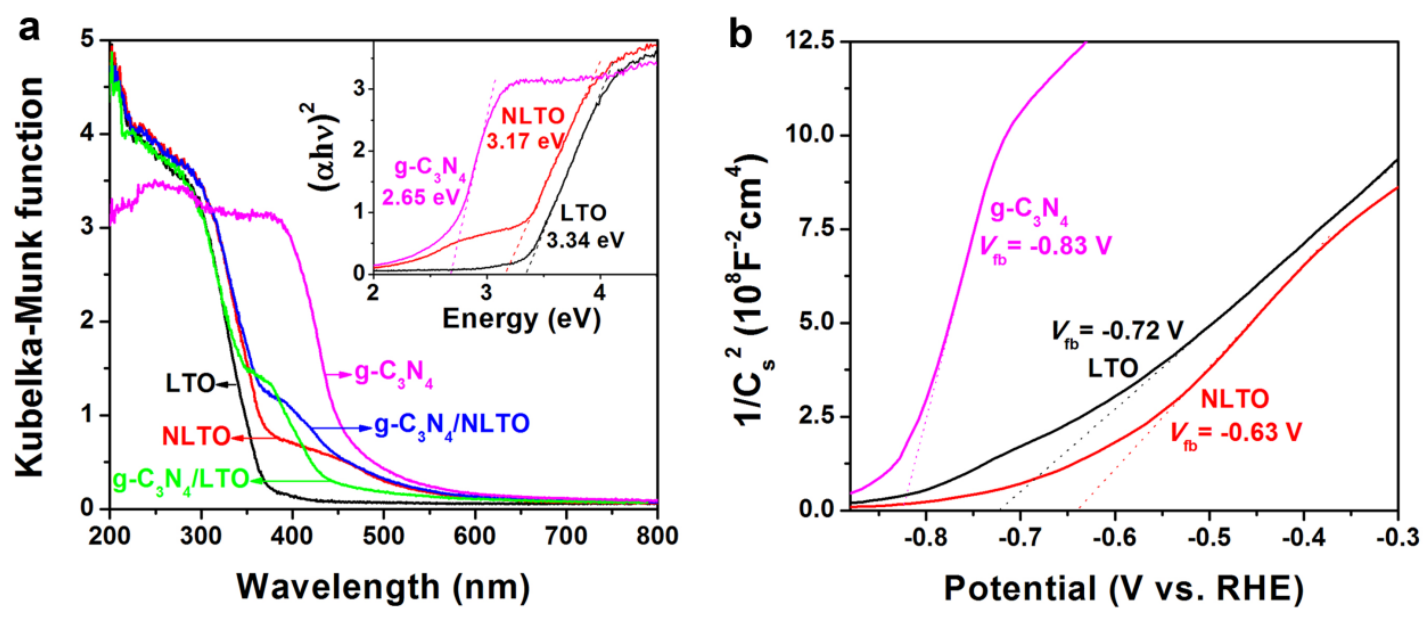

Fig. 4 

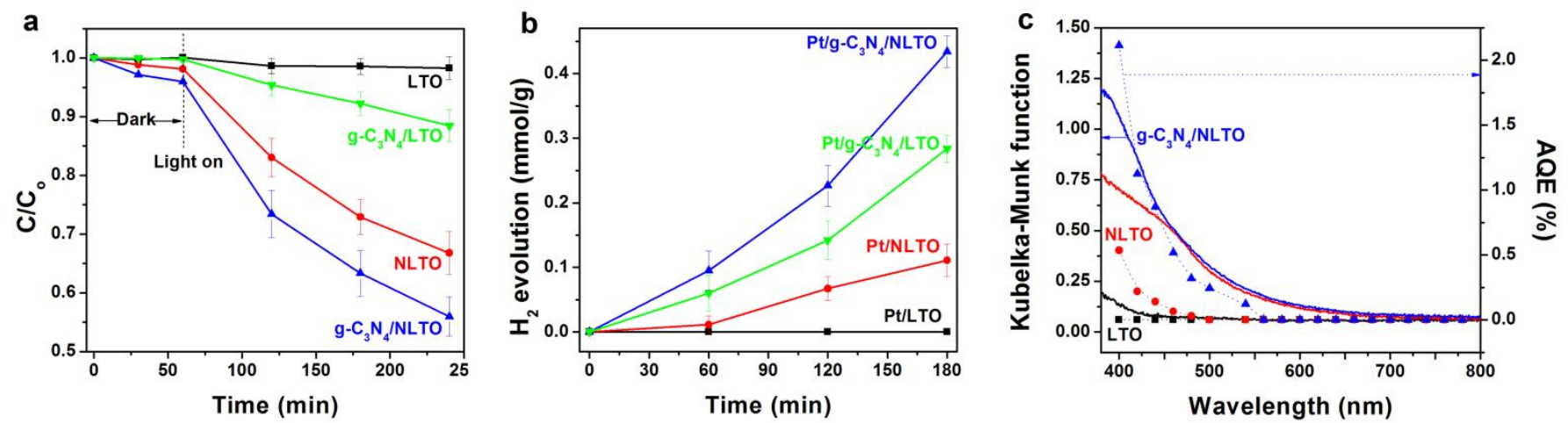

Fig. 5 

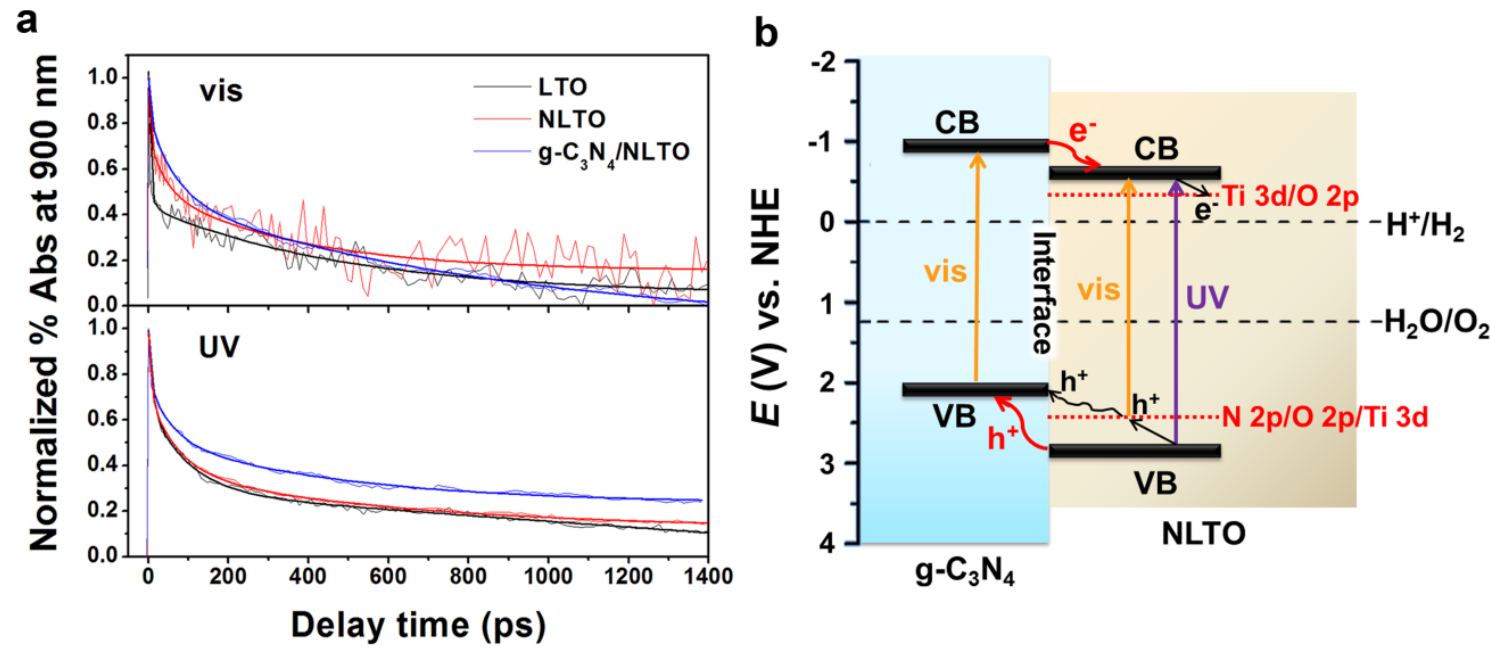

Fig. 6 
Table 1. Kinetic parameters of decays observed for LTO, NLTO, and $g-\mathrm{C}_{3} \mathrm{~N}_{4} / \mathrm{NLTO}$ under the visible or UV light irradiation

\begin{tabular}{|c|c|c|c|c|c|c|}
\hline \multirow{2}{*}{ Sample } & \multicolumn{3}{|c|}{ Visible light } & \multicolumn{3}{|c|}{ UV light } \\
\hline & $\tau_{1}(\mathrm{ps})$ & $\tau_{2}(\mathrm{ps})$ & $\tau_{3}(\mathrm{ps})$ & $\tau_{1}(\mathrm{ps})$ & $\tau_{2}(\mathrm{ps})$ & $\tau_{3}(\mathrm{ps})$ \\
\hline LTO & $2.2(49 \%)$ & $13(12 \%)$ & $353(39 \%)$ & $5.1(27 \%)$ & $55(34 \%)$ & $463(39 \%)$ \\
\hline NLTO & $2.9(40 \%)$ & $38(37 \%)$ & $864(23 \%)$ & $9.3(31 \%)$ & $74(50 \%)$ & $495(19 \%)$ \\
\hline $\mathrm{g}-\mathrm{C}_{3} \mathrm{~N}_{4} / \mathrm{NLTO}$ & $5.6(17 \%)$ & $68(35 \%)$ & $496(48 \%)$ & $6.8(31 \%)$ & $96(39 \%)$ & $627(30 \%)$ \\
\hline
\end{tabular}

